\title{
Time-dependent Effect of Graphene on the Microbial Activity of the Soil Under Single and Repeated Exposures
}

\author{
wenjuan liu \\ Shanxi University \\ yufeng guo \\ Shanxi University \\ guoli chai \\ Shanxi University \\ wenbo deng ( $\square$ dengwb@sxu.edu.cn ) \\ Shanxi University https://orcid.org/0000-0002-8659-3251
}

\section{Research Article}

Keywords: Graphene, Exposure scenarios, Soil bacterial community

Posted Date: January 5th, 2022

DOI: https://doi.org/10.21203/rs.3.rs-1049681/v2

License: (a) (i) This work is licensed under a Creative Commons Attribution 4.0 International License. Read Full License 


\section{Abstract}

Graphene (GR) has huge industrial and biomedical potential, and its adverse effect on soil microorganisms has been evaluated in ecotoxicological studies. These studies focus on a single exposure to GR, but repeated exposures are more likely to occur. In this study, we compared the impact of single and repeated exposures of GR on structure, abundance and function of soil bacterial community based on soil enzyme activity and high-throughput sequencing. The activities of urease and fluorescein diacetate esterase and alpha diversity demonstrate that repeated exposure to GR increase the diversity of soil bacteria. The PCoA and sample level clustering tree showed single exposure to GR after 4 days alter the soil bacterial community to some extent. During the entire incubation process, no matter what kind of exposure scenarios to GR, the majority of bacterial phylotypes remained unchanged except for Proteobacteria and Actinobacteria according to the relative abundance of phylotypes.

\section{Introduction}

Graphene (GR) is an atomically thin, tough two-dimensional, carbon-based materials consisting of $\mathrm{sp}^{2}$ hybridized carbon atoms(Rao et al. 2010). It is widely studied because of unique physicochemical properties that make them rapidly expanding in a large number of applications, including electronic devices, biosensors, water purification, biomedicine and drug delivery(Rasool et al. 2013; Xu et al. 2009). By 2023, the worldwide market volume of graphene composites will reach 1521 tons (Mendona et al. 2019). During the course of production and application, graphene will inevitably be discharged into the environment. The soil system may be the ultimate recipient for nanomaterials, including graphene. Model estimate that for carbon nanomaterials (for example, carbon nanotubes, graphene and fullerenes), with release rate of $0.004-1.6 \mu \mathrm{g} / \mathrm{kg}$ enter soil annually (Holden et al. 2014). Thus, graphene is nowadays considered as emerging contaminants in soil along with the concomitant need to understand its potential ecological effects.

In soil system, soil microbial communities are an indispensable part in nutrient cycling, the remediation of contaminated soil and especially sensitive indicator of soil's response to the environmental changes (Mukherjee and Acharya 2018; Liu et al.2015). However, few studies highlight the deleterious effect of graphene on soil communities to date. Ren et al(Ren et al. 2015) observed that graphene had a significant influence on the number of microorganisms and community structure in soil, and these effects are clearly related to the contact time graphene and microorganisms. Especially, when the concentration of graphene in the soil was extremely high, the bacteria population involved in nitrogen biogeochemical cycles and the degradation of organic compounds were significantly reduced. And some studies stated that low concentration of graphene oxide could promote the growth of microorganism, which might due to the increase of the cell attachment and proliferation(Zhao et al. 2020). Furthermore, Lanphere et al (Lanphere et al. 2013) pointed out that the rate of graphene migration in soil decreased with increasing ion intensity. And related studies showed that in addition to the ion concentration, the inorganic matter and organic matter in the soil will also affect the mobility and bioavailability of graphene(Lanphere et al. 2013). In brief, through the above studies, we can clearly notice that the effect's degree of graphene on 
soil microbial communities depends on the concentration of graphene, the length of exposure time and the external environment.

In these studies, graphene was performed as a single homogeneous and acute exposure to soil. This exposure model may lead to more resistant microbial communities to graphene perturbation if sensitive communities are replaced by resistant communities (Simonin et al. 2016). However, given the practical environmental contamination, graphene is more likely to be released into the soil chronically through repeated application. Up to date, little information is available to illustrate the changes in soil microbial activity affected by GR under repeated exposures. This chronic exposure is likely to have more detrimental effects. It is necessary to consider exposure modes in the experiment, which is crucial for assessing the microbial responses accurately to the effect of graphene thus the variations of terrestrial ecosystem service and stability. Furthermore, a comparative study between single and repeated exposures to graphene on soil bacterial communities can provide a reference for future studies that which exposure scenario should be paid more intensive attention.

In this context of lab-scale experiment, soil was contaminated using three exposure scenarios (one, two and three applications), resulting in the same total graphene concentrations (300mg of graphene $\mathrm{kg}^{-1}$ of soil). After the last application, the contaminated soil was incubated for 2 months to evaluate the impact of graphene on soil bacterial communities. The changes in the bacterial abundance and the structure of the soil bacterial community were determined by high-throughput sequencing of $16 \mathrm{~S}$ rRNA genes. And the effect of graphene on microbial function were evaluated by soil enzyme activities, which are considered as sensitive indicators of changes in the microbial community under nanomaterials stress. This work conducts a comparative study between single and repeated exposures to graphene on soil bacterial communities, which can provide a reference for future studies that exposure scenario should be paid more intensive attention.

\section{Material And Methods}

\subsection{Graphene and soil}

Graphene (500-1000 $\mathrm{m}^{2} / \mathrm{g}$ specific surface area,0.5-3.74nm thickness, purity $>95 \%$ wt.) was purchased from Shanghai Aladdin Biochemical Technology Co., Ltd. And other reagents were obtained from Tian-jin Reagent Factory. All reagents of analytical grade were used without prior purification. The solutions used in this work were prepared with deionized distilled water.

The surface soil sample were collected from cropland at Dongyang Agroecological Experimental station of Shanxi Academy of Agricultural Sciences $\left(112^{\circ} 40^{\prime} \mathrm{E}, 37^{\circ} 32^{\prime} \mathrm{N}\right)$ at a depth of $5-10 \mathrm{~cm}$. Large root fragments and particles were removed, and the soil sample was sieved to $2 \mathrm{~mm}$. The soil was stored in polyethylene bags at $4^{\circ} \mathrm{C}$ until analysis. The characteristics of the soil were as shown: $\mathrm{pH}, 8.42$; organic matter, $6.92 \mathrm{~g} / \mathrm{kg}$; total nitrogen, $0.81 \mathrm{~g} / \mathrm{kg}$; available phosphorus, $4.43 \mathrm{mg} / \mathrm{kg}$; available potassium, 131.52 $\mathrm{mg} / \mathrm{kg}$. 


\subsection{Exposure experiment}

Prior to the incubation experiment, the soil was incubated at $25^{\circ} \mathrm{C}$ for three days to revive the soil microorganism. $100 \mathrm{~g}$ soil samples were weighted into $500 \mathrm{~mL}$ triangular flasks for each treatment. For the three different exposure scenarios, specific amount of graphene was added to the soil: $30 \mathrm{mg}$ for the single-exposure experiment, $15 \mathrm{mg}$ for the two-exposure experiment, $10 \mathrm{mg}$ for the three-exposure experiment, resulting in the same final graphene concentration of $300 \mathrm{mg} / \mathrm{kg}$ (Table 1). Due to the real environmental concentration of GR is still unknown, this concentration was choose based on the model estimation (Gottschalk et al. 2013). Sterilized water was added weekly to adjust the soil moisture to 13.6 $\%$, which was detected by drying for $8 \mathrm{~h}$ at $105^{\circ} \mathrm{C}$. The successive addition of GR was separated by a 15day delay, during which the soil samples were incubated at $25^{\circ} \mathrm{C}$ in the dark. And for each separate subsample, they were collected after 4, 21 and 60 days of incubation following the last application of GR to soil (Table 2). Soil without GR was used as the control. All treatments were performed with three replicates.

\subsection{Soil enzyme activities}

The enzyme activities of urease and FDA esterase for all soil subsamples were determined according to the previous methods (Liu et al. 2015). The enzyme assays were carried out in triplicate for each treatment.

\subsection{DNA extraction and high-throughput sequencing}

For all the cultured soil samples, $0.2 \mathrm{~g}$ soil was used to extract the total genomic DNA with a E.Z.N.A. Soil DNA Kit (Omega, USA). The concentration of the DNA was measured using a Qubit 3.0 (life, USA) to ensure that adequate amounts of high-quality genomic DNA had been extracted.

The V3-V4 region of the bacterial 16S rRNA genes was amplified using KAPA HiFi Hot Start Ready Mix (2x) (TaKaRa Bio Inc., Japan). The universal bacterial 16S rRNA gene amplicon PCR primers were used: the primer pair 341F (CCTACGGGNGGCWGCAG) and 805R (GACTACHVGGGTATCTAATCC). The reaction was set up as follows: microbial DNA (10 ng/ $\mu \mathrm{L}) 2 \mu \mathrm{L}$; amplicon PCR forward primer $(10 \mu \mathrm{M}) 1 \mu \mathrm{L}$; amplicon PCR reverse primer $(10 \mu \mathrm{M}) 1 \mu \mathrm{L} ; 2 \times$ KAPA HiFi Hot Start Ready Mix $15 \mu \mathrm{L}$ (total $30 \mu \mathrm{L}$ ). The plate was sealed and PCR performed in a thermal instrument (Applied Biosystems 9700, USA) using the following program: 1 cycle of denaturing at $95^{\circ} \mathrm{C}$ for $3 \mathrm{~min}$, first 5 cycles of denaturing at $95^{\circ} \mathrm{C}$ for $30 \mathrm{~s}$, annealing at $45^{\circ} \mathrm{C}$ for $30 \mathrm{~s}$, elongation at $72^{\circ} \mathrm{C}$ for $30 \mathrm{~s}$, then 20 cycles of denaturing at $95^{\circ} \mathrm{C}$ for $30 \mathrm{~s}$, annealing at $55^{\circ} \mathrm{C}$ for $30 \mathrm{~s}$, elongation at $72^{\circ} \mathrm{C}$ for $30 \mathrm{~s}$ and a final extension at $72^{\circ} \mathrm{C}$ for $5 \mathrm{~min}$. The PCR products were checked using electrophoresis in $1 \%(\mathrm{w} / \mathrm{v})$ agarose gels in TBE buffer (Tris, boric acid, EDTA) stained with ethidium bromide (EB) and visualized under UV light. The AMPure XP beads were used to purify the free primers and primer dimer species in the amplicon product. Samples were delivered to Sangon BioTech (Shanghai) for library construction using universal Illumina adaptor and index. Before sequencing, the DNA concentration of each PCR product was determined using a Qubit $\circledast 2.0$ Green double-stranded DNA assay and it was quality controlled using a bioanalyzer (Agilent 2100, USA). 
Depending on coverage needs, all libraries can be pooled for one run. The amplicons from each reaction mixture were pooled in equimolar ratios based on their concentration. Sequencing was performed using the Illumina MiSeq system (Illumina MiSeq, USA), according to the manufacturer's instructions.

After sequencing, data were collected as follows:(1) The two short Illumina readings were assembled by PEAR (v0.9.6) soft-ware according to the overlap and fastq files were processed to generate individual fasta and qual files, which could then be analyzed by standard methods. (2) Sequences containing ambiguous bases and any longer than 480 base pairs (bp) were dislodged and those with a maximum homopolymer length of $6 \mathrm{bp}$ were allowed. And sequence short than 200bp were removed. (3)All identical sequences were merged into one.(4)Sequences were aligned according to a customized reference database.(5) The completeness of the index and the adaptor was checked and removed all of the index and the adaptor sequence.(6) Noise was removed using the Pre.cluster tool. Chimeras were detected by using Chimera UCHIME. All the software was in the mothur package. We submitted the effective sequences of each sample to the RDP Classifier again to identify bacterial sequences. The modified pipeline is described on the mothur website. Finally, all effective bacterial sequences without primers were submitted for downstream analysis.

\subsection{Statistical analysis}

Alpha diversity was used to measure the abundance and diversity of the microbial community. The Shannon index was used to describe the soil bacterial alpha diversity, due to it evaluates both evenness and richness. The Bray-Curtis distance was calculated to determine the difference in bacterial beta diversity. And the Principal coordinate analysis (PCoA) and sample clustering tree based on UnweightedUnifrac distance were conducted to visualize the control-to-treatment dissimilarity in microbial community structure. A Venn diagram was constructed to count intuitively the unique and shared number of operational taxonomic units (OTUs) between the control and treated samples. The relative abundance was summarized at phylum level to compare the community structure among the control and the treatment.

\section{Results And Discussion}

\subsection{Effect of GR on soil enzyme activities}

The soil enzyme activities are considered as excellent indicators of changes of soil microbial activity under GR stress. Fig1 summarize the enzyme activity of soil exposure to GR through different adding methods. A decrease in the enzyme activities of urease and FDA esterase for single exposure after 4 days of incubation following the last application of GR to soil, which is consistent with the studies of some studies (Ren et al. 2015). Due to the high surface-to-volume and low homeostatic capacity of microorganisms, their activity decreased a little under high concentration of GR exposure for a short time(Shrestha et al. 2013). However, up to 21 days, the enzyme activities of these two enzymes for repeated exposures were increased by approximately $20 \%$, and the enzyme activities under the two exposures increased more than under three exposures. This is probably because that the acute and high 
concentration of GR exposure repressed the growth of some soil microorganisms which produced these enzymes, and the chronic and low concentration of GR proliferated to a much greater extent than the bacteria that were inhibited. As the increase of exposure time, the gradual growth of the relevant microorganisms narrowed the gap between the single and repeated exposures after exposure for a longer time. A possible explanation is that the soil microbes start to tolerate and adapt the inhibitor after some time which is consistent with the environ-mental change by transformation of metabolism (Simonin et al. 2015). In the course of the whole experiment, the change of urease activities under GR stress for different exposure time were greater than that of the FDAE activities. Urease is a representative extracellular enzyme and the key source of bacterial nitrogen, appears to be more sensitive to pollution than FDAE activities which represent overall microbiological activity of soil. And with the extending of incubation time, GR will likely increase soil nitrogen cycling, and urease activities significantly increased in the soil community.

\subsection{Effect of GR on soil bacterial community alpha diversity}

Based on high-throughput sequencing, the alpha diversity (Shannon index) of different treated soil were displayed in Fig.2. The alpha diversity at the beginning of exposure to GR through different methods was almost identical because the bacterial community was from the same soil sample. Although the final concentration of GR was the same, alpha diversity followed an order of GR31>GR21>GR11 after 4 days incubation. This result demonstrated that high concentration of GR exposure for the short term decreased the diversity within a specific bacterial community, and in the low concentration range, soil microbial diversity increased, so the diversity under repeated exposures increased more than under single exposure after 4 days incubation. When the incubation time reached 21 days, the alpha diversity of repeated exposures soils reached a maximum, which indicated that the repeated exposures exhibited pronounced increased after a relatively longer time. When the incubation time was prolonged to 60 days, the alpha diversity decreased. This is due to that the relatively tolerant and resistant species became more abundant and occupied the niche of the sensitive species that were eliminated, leading to a lesser diverse (Song et al. 2018). These results are consistently with previous work that the soil bacterial alpha diversity under exposure to GR gradual decrease when the exposure time was longer than 60 days, regardless of the GR concentration (Wu et al. 2020). Overall, the results suggested that with the extension of exposure time the repeated exposures have more positive effect on the soil alpha diversity.

\subsection{Effect of GR on soil bacterial community Beta diversity}

In order to compare similarities of soil bacterial community compositions under different exposure method, the Bray-Curtis distance of soil samples were compared relative to control. From Fig.3, the repeated exposures of GR played a stronger effect on soil bacterial communities as compared to the single exposures after 4 days incubation, while this effect became weak gradually with the extension of incubation time. The principal co-ordinates analysis (PCoA) based on Unweighted-UniFrac distance which incorporates phylogenetic distance into relative abundance measurement was also conducted to study the difference of soil bacterial community. The PCoA results showed that, the soil bacterial community 
after exposure to GR in a short-term time were totally separated from the control, especially for the soil under single exposure. And the soil bacterial communities changed constantly with the growth of culture time, and the distinction between the single exposure and repeated exposures got smaller. In addition, after a longer period, the sample from the control group, the repeated GR exposure were clustered with single GR exposure, suggesting similar microbial community structures. Furthermore, after 60 days incubation, the soil bacterial communities of all the soil samples were totally different form that of the initial samples. And adonis analysis on the bacterial community further confirmed that the difference in soil bacterial communities under different incubation time to GR was significant $\left(R^{2}=0.573, p=0.001\right)$.

Different exposure model induced certain shifts in the soil bacterial community

composition, some core taxa still remained under all treatments. Via high-throughput sequencing, the Venn diagrams showed that a total of 2334 OTUs, 29650TUs and 2910 OTUs were detected under the different exposure time. The $10.92 \% 6.35 \%, 11.7 \%$ and $12.68 \%$ of all OTU were specific to control, 1 exposure, 2 exposures and 3 three exposures after 4 days incubation. However, as the incubation time lengthened to 60 days, these data were $9.68 \% 8.05 \%, 9.43 \%$ and $9.87 \%$, respectively. These results indicated that the in the short term, adding a certain amount of graphene to a soil at a time may inhibit the growth of some unique species. Together, both beta diversity and core taxa analysis suggest that, yet in the long run no matter what exposure scenario is, and GR could promote soil bacteria to a certain extent.

\subsection{Effect of GR on soil bacterial populations at phylum level}

To further identify the changes in the bacterial community of soil exposure to GR through different adding method, the sample level clustering tree and the relative abundance $\mathbb{R} A \llbracket$ of phylotypes was summarized at the phylum level based on the pyrosequencing data. According to the Figure 4A, the soil bacterial communities of single exposure were obviously different from that of repeated exposures when the incubation time was lower than 21 days, and the difference grow small with the extending of incubation time. And the soil bacterial community of all the soil including control changed when the incubation time reached 60 days according to the sample level clustering tree. Figure 4B revealed that there was high bacterial diversity in all treated soil and control samples, and $85 \%$ of the total bacterial counts could be represented by eight predominant bacterial phyla among which Proteobacteria, Acidobacteria, Planctomycetes, Actinobacteria, and Bacteroidetes are the dominant phyla in agricultural soils(Buckley and Schmidt 2003). The Proteobacteria is the most predominant phylum in the soil bacterial community (33.62\%-52.01\%), which consists of a majority of Gram-negative bacteria(MaiProchnow et al. 2016). And the RA of Proteobacteria in soil under repeated exposure increased more than that under single exposure after 4 days incubation, the gap had been narrowed with the extension of time. Normally, the Gram-negative bacteria have a higher tolerance to external stress than Gram-positive bacteria because of their outer membrane and cell wall(Premanathan et al. 2011). And the phylum Proteobacteria could participate in degradation of carbonaceous compounds(Cebron et al. 2008; Spain et al. 2009). So, an increase in RA of Proteobacteria under multiple exposure to GR reflected its high 
resistance and high carbon mineralization rate in soil. It is obvious that Proteobacteria and Acidobacteria were the primary phylum under a short period ( 4 and 21 days). The acidobacteria and actinobacteria were slightly reduced under GR exposure compared to control, and the rest of phylum did not change significantly under any of the GR exposure way, except for these three phyla. Intriguingly, with the extension of time, up to 60 days, for all the soil sample, not only the Proteobacteria increased, but also the actinobacteria increased for all the soil samples. The phylum Actinobacteria has the potential to degrade lignocellulose; some tentative cellulose degrading enzymes were found in Actinobacteria genomes( Tetrovsky et al. 2014). So, the lignocellulose degradation rate for all soil increased because of the potential to degrade lignocellulose of phylum Actinobacteria. Based on sample level clustering tree and the relative abundance $\mathbb{R} A$ \of phylotypes, it could be inferred that graphene changed the soil bacterial community to a certain extent and the changes became weaker or disappeared with extending of exposure time no matter what exposure scenarios were.

\section{Conclusion}

In summary, our work provides useful information for comprehensive understanding the ecotoxicological effect of GR on soil bacterial communities under different exposure scenarios. The activities of urease and fluorescein diacetate esterase demonstrate that the single exposure play a negative effect on the structure, function and diversity of the soil bacterial community, particularly during early stages of incubation. The richness and diversity indices of bacteria community in soil under repeated exposures to GR increase more than under single exposure to GR, implying that different exposure scenarios to GR in soil exhibit diverse effect on soil bacteria community. To conclude, the repeated exposures to GR promotes the growth of microorganism in soil sample and that a chronic exposure to GR is more helpful for soil bacteria than a single exposure.

\section{Declarations}

\section{Declaration of Competing Interest}

The authors declare no competing financial interest.

\section{Acknowledgement}

This work was financially supported by the National Natural Science Foundation of China (41907277), and Scientific and Technological Innovation Programs of Higher Education Institutions in Shanxi \2019L0038 and 2019L0071『and the Shanxi Province Hundred Talents Project.

\section{References}

Buckley DH, Schmidt TM (2003) Diversity and dynamics of microbial communities in soils from agroecosystems. Environ Microbiol 5(6):441-452 
Cebron A, Norini MP, Beguiristain T, Leyval C (2008) Real-Time PCR quantification of PAH-ring hydroxylating dioxygenase (PAH-RHDa) genes from Gram positive and Gram negative bacteria in soil and sediment samples. J Microbiol Meth 73(2):148-159

Gottschalk F, Sun TY, Nowack B (2013) Environmental concentrations of engineered nanomaterials: Review of modeling and analytical studies. Environ pollut 181:287-300

Holden PA, Klaessig F, Turco RF, et al (2014) Evaluation of Exposure Concentrations Used in Assessing Manufactured Nanomaterial Environmental Hazards: Are They Relevant? Environ Sci Technol 48(18):10541-10551

Lanphere JD, Walker SL (2013) Effects of solution chemistry on the transport of graphene oxide in saturated porous media. Environ Sci Technol 47(9):4255-4261

Mai-Prochnow A, Clauson M, Hong JM, Murphy ABJSR (2016) Gram positive and Gram negative bacteria differ in their sensitivity to cold plasma. Sci Rep 6(1):38610

Mendona MCP, Rodrigues NP, Jesus MB, Amorim MJB (2019) Graphene-Based Nanomaterials in Soil: Ecotoxicity Assessment Using Enchytraeus crypticus Reduced Full Life Cycle. Nanomaterials 9(6)

Premanathan M, Karthikeyan K, Jeyasubramanian K, Manivannan G (2011) Selective toxicity of ZnO nanoparticles toward Gram-positive bacteria and cancer cells by apoptosis through lipid peroxidation. Nanomed-Nanotechnol 7(2):184

Rao CNR, Sood AK, Subrahmanyam KS, Govindaraj A (2010) Graphene: The New Two-Dimensional Nanomaterial. Angew Chem Int Edit 40(52)

Rasool HI, Ophus C, Klug WS, Zettl A, Gimzewski JK (2013) Measurement of the intrinsic strength of crystalline and polycrystalline graphene. Nat Commun. 4

Ren WJ, Ren GD, Teng Y, Li ZG, Li LN (2015) Time-dependent effect of graphene on the structure, abundance, and function of the soil bacterial community. J Hazard Mater 297(30):286-294

Shrestha B, Acosta-Martinez V, Cox SB, Green MJ, Li S, Canas-Carrell (2013) An evaluation of the impact of multiwalled carbon nanotubes on soil microbial community structure and functioning. $J$ Hazard Mater 261(15):188-197

Simonin M, Martins JMF, Uzu G, Vince E, Richaume A (2016) Combined study of titanium dioxide nanoparticle transport and toxicity on microbial nitrifying communities under single and repeated exposures in soil columns. Environ Sci Technol 50(19):10693-10699

Simonin M, Richaume A] (2015) Impact of engineered nanoparticles on the activity, abundance, and diversity of soil microbial communities: a review. Environ Sci Pollut R 22(18):13710-13723 
Song JF, Duan CW, Sang Y, Wu SP, Ru, JX, Cui XY (2018) Effects of Graphene on Bacterial Community Diversity and Soil Environments of Haplic Cambisols in Northeast China. Forest 9(11):677

Spain AM, Krumholz LR, Elshahed MS (2009) Abundance, composition, diversity and novelty of soil Proteobacteria. ISME Journal 3(8):992-1000

Tetrovsky T, Steffen KT, Baldrian P (2014) Potential of Cometabolic Transformation of Polysaccharides and Lignin in Lignocellulose by Soil Actinobacteria. Plos One 9(2):e89108

Liu WJ, Yao J, Chai, HK, et al (2015) Concentration-dependent effect of photoluminescent carbon dots on the microbial activity of the soil studied by combination methods. Environ Toxicol Pharmacol 39(2), 857863.

Wu F, Jiao S, Hu J, Wu XY, et al (2020) Stronger impacts of long-term relative to short-term exposure to carbon nanomaterials on soil bacterial communities. J Hazard Mater 410124550

Xu X, Qin J, Zhen L (2009) Research Advances of Graphene. Prog Chem 21(12):2559-2567 21(12):25592567

Zhao DQ, Fang ZW, Tang YH, Tao J (2020) Graphene Oxide as an Effective Soil Water Retention Agent Can Confer Drought Stress Tolerance to Paeonia ostii without Toxicity. Environ Sci Technol 54(13)82698279

\section{Tables}

Table 1. The experiment layout

\begin{tabular}{|llll|}
\hline Treatment & $\begin{array}{l}\text { Single exposure to GR } \\
\left(\mathrm{mg} \cdot \mathrm{kg}^{-1}\right)\end{array}$ & $\begin{array}{l}\text { Two exposures to GR } \\
\left(\mathrm{mg} \cdot \mathrm{kg}^{-1}\right)\end{array}$ & $\begin{array}{l}\text { Three exposures to GR } \\
\left(\mathrm{mg} \cdot \mathrm{kg}^{-1}\right)\end{array}$ \\
\hline First day & 300 & 150 & 100 \\
\hline 15 days later & 0 & 150 & 100 \\
\hline 30 days later & 0 & 0 & 100 \\
\hline
\end{tabular}

Table 2 is not available with this version

\section{Figures}



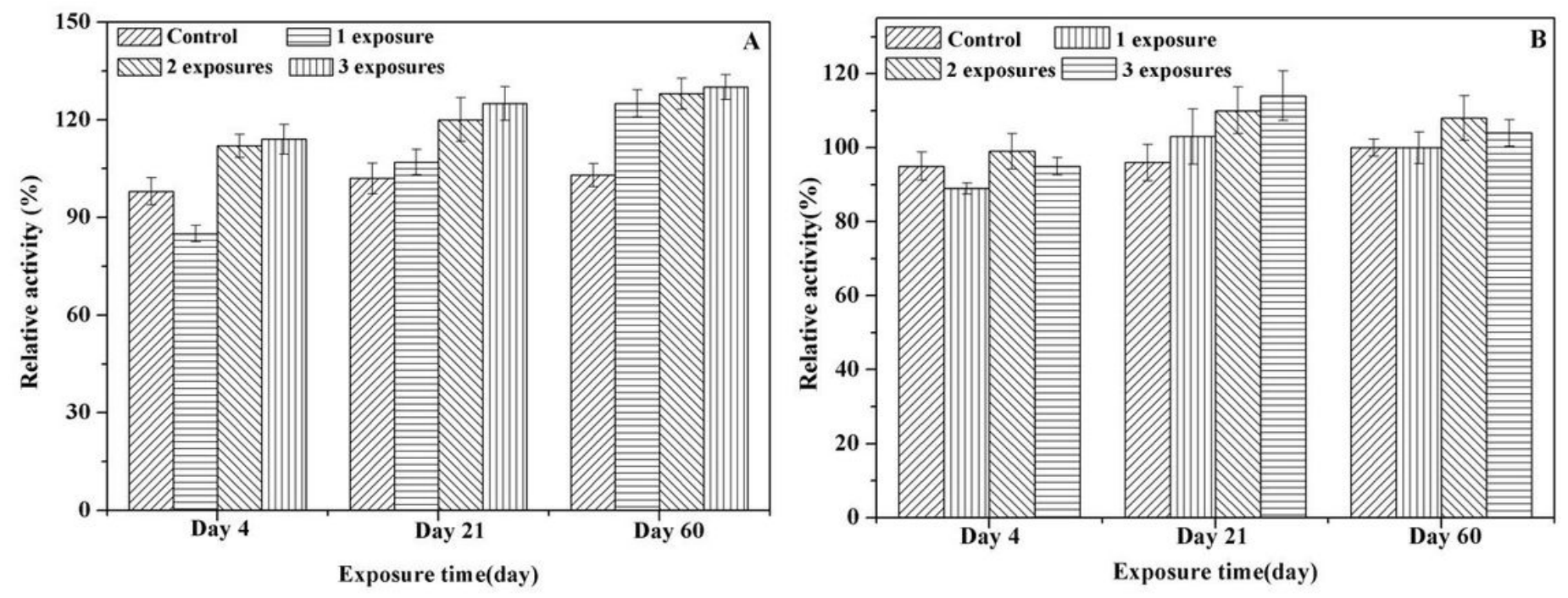

Figure 1

Urease (A) and FDAE (B) activity of soil exposed to GR under three different exposure scenarios. The bars indicate the standard deviations. 


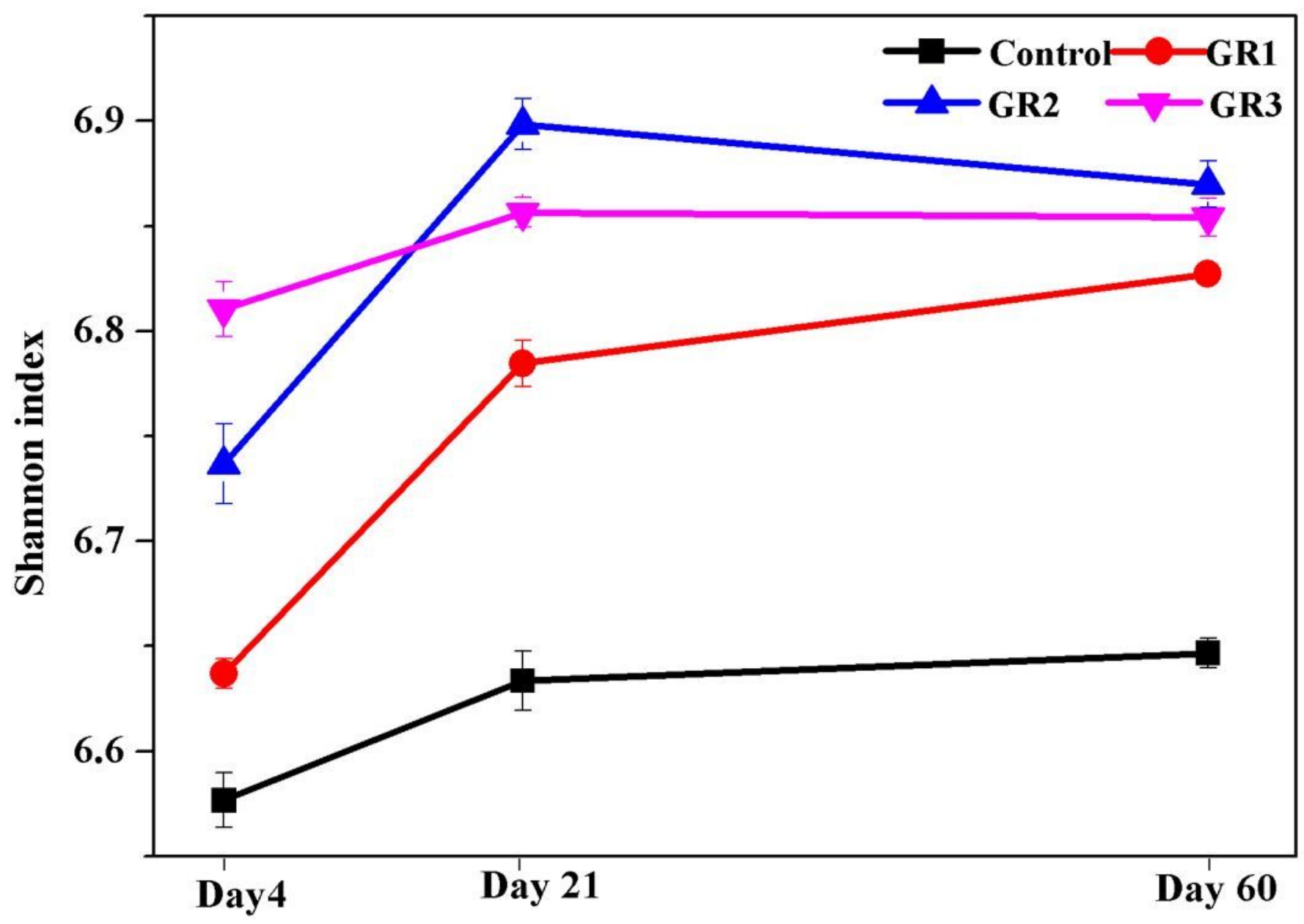

Incubation days

Figure 2

Temporal trends of alpha diversity of soil bacterial communities exposed to GR under three different exposure scenarios. The bars indicate the standard deviations. (GR1: 1exposure; GR2: 2 exposures; GR3: 3 exposures) 



Figure 3

Bray-Curtis distances between each treatment and the control(A) PCoA of Unweighted-UniFrac distance(B) of soil bacterial community exposed to GR under three different exposure scenarios (C: control; GR1: 1exposure; GR2: 2 exposures; GR3: 3 exposures). The percentage of the total variance explained by each axis is shown. 



\section{Figure 4}

Venn diagrams of common and unique OTUs in soil bacterial communities under three different exposure scenarios (C: control; GR1: 1 exposure; GR2: 2 exposures; GR3: 3 exposures).

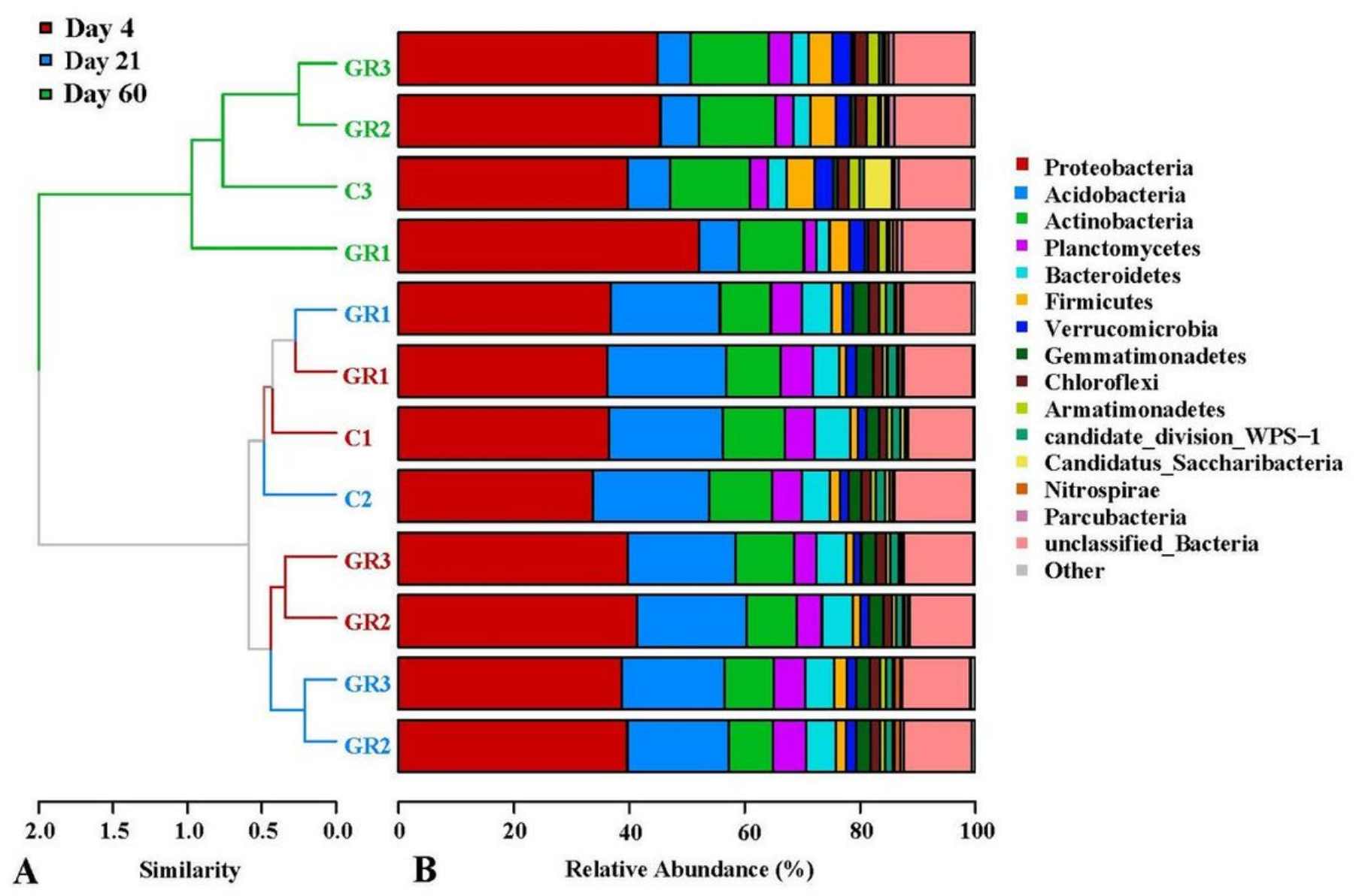

\section{Figure 5}

Sample level clustering tree (A) and the relative abundance『RA『of phylotypes(B) in soil bacterial communities under three different exposure scenarios (C: control; GR1: 1exposure; GR2: 2 exposures; 
GR3: 3 exposures).

Page 15/15 